\section{Extensión universitaria y cooperativas: prácticas y perspectivas de una década (Argentina, 1958-1968) ${ }^{1}$}

\section{Mirta Vuotto}

Centro de Estudios de Sociología del Trabajo.

Universidad de Buenos Aires, Argentina.

mirta.vuotto@fce.uba.ar,mirta.vuotto@gmail.com
Investigación y extensión universitaria / Perspectivas
RECEPCIÓN: 29/05/18

ACEPTACIÓN FINAL: 28/06/18

\section{Resumen}

La extensión universitaria ha sido objeto de algunas consideraciones acerca de su naturaleza y el papel que debe ocupar entre las principales funciones institucionales. Sin embargo, pocos trabajos han planteado una reflexión sobre su impacto a la luz de las realizaciones ocurridas en el campo específico de las cooperativas.

El artículo se propone definir y analizar los principales rasgos de un programa de extensión impulsado por la Universidad de Buenos Aires entre 1958-1968 para propiciar espacios de promoción del apoyo y asistencia de la actividad de las cooperativas. Se introducen antecedentes sobre el origen de la extensión universitaria para caracterizar el programa y mencionar la contribución al mismo por parte de la Facultad de Ciencias Económicas. Como conclusión, se plantea una reflexión sobre las modalidades de los programas de extensión para desarrollar estrategias efectivas que permitan mejorar el compromiso de las universidades en una perspectiva de largo plazo.

Palabras clave: cooperativas, extensión universitaria, programas de extensión, comunidad
University extension and cooperatives: practices and perspectives over a decade (Argentina, 1958-1968)

\section{Abstract}

University extension has been the object of some considerations about its nature and the role it should take among the main institutional functions. However, few scholars have given due consideration to its impact, considering the achievements that have taken place in the specific field of cooperatives.

This article aims to analyze the main features of an extension program developed by the University of Buenos Aires between 1958-1968 to foster spaces for the promotion of support and assistance to cooperative entities. Antecedents about the origin of the university extension experience are introduced to characterize the program and to mention contributions made by the Faculty of Economics Sciences.

The article concludes with a discussion on the possible directions that the extension programs could take in terms of effective strategies to improve the commitment of universities in a long-term perspective.

Keywords: cooperatives, university extension, extension programs, community
Extensão universitária e cooperativas: práticas e perspectivas ao longo de uma década (Argentina, 1958-1968)

\section{Resumo}

A extensão universitária tem sido objeto de diferentes considerações sobre sua essência e o papel que deve ocupar entre as principais funções institucionais. Entretanto, são poucos os trabalhos que realizaram uma reflexão sobre seu impacto à luz das realizações ocorridas no campo específico das cooperativas.

Este artigo tem por objetivo analisar as principais caraterísticas de um programa de extensão desenvolvido pela Universidade de Buenos Aires entre 1958-1968, para propiciar espaços de promoção do apoio e assistência da atividade das cooperativas. São apresentados alguns antecedentes sobre a origem da extensão universitária na universidade, para caracterizar o programa e mencionar a contribuição da Faculdade de Ciências Econômicas.

A título de conclusão, propõe-se uma reflexão sobre os programas de extensão para desenvolver estratégias efetivas para melhorar o compromisso das universidades em uma perspectiva de longo prazo.

Palavras-chave: cooperativas, extensão universitária, programas de extensão, comunidade

Para citación de este artículo: Vuotto, M. (2018). Extensión universitaria y cooperativas: prácticas y perspectivas de una década (Argentina 1958-1968). +E: Revista de Extensión Universitaria, 8(8), enero-junio, 44-60. doi: 10.14409/extensión.v8i8.Ene-Jun.7717.

1) La autora agradece las valiosas sugerencias de los evaluadores anónimos. 


\section{Introducción}

La extensión universitaria ha sido objeto de distintas consideraciones que focalizan -por lo general- en el papel que debe ocupar entre las principales funciones institucionales y visualizan la responsabilidad de la universidad pública como agente de transformación en la promoción del conocimiento dirigido a distintos actores sociales. Los programas de extensión en el ámbito universitario tienen entre sus propósitos estimular el desarrollo de competencias ciudadanas que fomenten la cooperación y permitan eliminar los obstáculos de carácter económico, social, cultural y políticos que limitan la colaboración entre personas, grupos e instituciones.

Aunque en distintos ámbitos institucionales se han considerado los compromisos públicos que supone la extensión, analizando su pertinencia, relevancia social y sectores y problemáticas a las que intenta responder, no se han identificado trabajos que hayan planteado una reflexión sobre su impacto a la luz de realizaciones en el campo específico del cooperativismo argentino.

El artículo se propone caracterizar la naturaleza y proyección de los programas de extensión impulsados por la Universidad de Buenos Aires (UBA) entre 1958-1968 al propiciar, entre otros, un espacio de promoción y acompañamiento de la actividad de las cooperativas. El texto considera algunos antecedentes sobre el origen de la extensión universitaria y su vínculo con las cooperativas para referir específicamente a los alcances del programa desarrollado en la UBA durante esa década y el papel de la Facultad de Ciencias Económicas.

Como conclusión, se reflexiona sobre el alcance de dos modalidades de extensión que han incorporado estrategias diversas y acciones diferenciadas en cuanto a contenidos específicos y componentes pedagógicos vinculados con el cooperativismo.

\section{Algunos antecedentes sobre la extensión universitaria}

Uno de los primeros antecedentes documentados sobre la naturaleza de la extensión universitaria y su promoción en el siglo XIX fue la carta que James Stuart ${ }^{2}$ dirigió a los miembros de la Universidad de Cambridge (Stuart, 1871). En ella destacó la importancia de llevar los cursos de la universidad a regiones alejadas de la institución para difundir el conocimiento entre vastos sectores sociales imposibilitados de alcanzarlo. Mencionaba la importancia de acceder a los conocimientos que brindase el docente, dirigiéndose especialmente a las clases que más lo desearan y tuviesen imposibilidad para ello (Stuart:1). ${ }^{3}$ Un aspecto destacado dentro del sistema concebido por Stuart correspondía a los tiempos que podían asignar los docentes a sus tareas y los presupuestos requeridos para financiar las actividades de extensión. Este aspecto se ilustraba refiriendo a una conversación mantenida con algunos miembros del Comité de Educación de la Sociedad de Pioneros Equitativos de Rochdale, que le

2) James Stuart fue un activista destacado, educador y promotor de la extensión universitaria. Egresado del Madras College y la Universidad de St. Andrews, donde se graduó en 1861, realizó estudios en el Trinity College desempeñándose como tutor asistente en la Universidad de Cambridge. En 1867 dictó conferencias sobre el tema de la extensión en instituciones como England Council for Promoting the Higher Education of Women y London Society for the Extension of University Education.

3) La carta de Stuart menciona que el sistema instaurado en 1867, de promoción de la educación superior para mujeres, fue adoptado desde entonces en Edimburgo, Londres, y en cerca de veinte ciudades del Reino Unido, extendiéndose luego en América del Norte. 
habían expresado que "el organismo estaría dispuesto a garantizar £50 a £100 anuales para un plan de este tipo en su ciudad" (5-7).

El enfoque integrador en materia de estrategia y política extensionista, al valorizar un tipo de formación de carácter práctico, permitió trascender la tradición teórica de Cambridge, e impulsar la creación de un programa formal de cursos de extensión. La iniciativa de Stuart permitió promover el papel de la extensión y alcanzar un gran impacto en audiencias integradas por maestros, artesanos y cooperativistas

"debido al entusiasmo que suscitó en las clases trabajadoras, del que numerosas evidencias atestiguan su sinceridad. En más de un centro industrial, los sindicatos, las sociedades cooperativas y todas las asociaciones obreras han suscrito su ayuda a los miembros del Comité Local de Extensión para completar los fondos requeridos para organizar uno o varios cursos". (Leclerc, 1892:34)

Esta iniciativa se fue transformando al extenderse al continente europeo para llegar a los Estados Unidos y cristalizar en la creación, en 1890, de la American Society for the Extension of University Teaching, institución que destacó su importancia, distinguiéndola por hacer de la educación uno de los intereses permanentes de la vida, junto con la religión y la política. Desde esta perspectiva, la extensión fue considerada como "el cambio silencioso que ha reclamado la educación superior, la educación de la vida que pertenece a la enseñanza universitaria y la reivindicación del patrimonio de cada buen ciudadano" (Moulton, 1890:6).

Los testimonios sobre las distintas realizaciones evidenciaron la forma en que la acción extensionista alcanzó ámbitos diversos por estar concebida "para todas las clases de personas: ricos y pobres, empleado y empleador, obrero y capitalista, hombres y mujeres, educado y sin educación" (James, 1895:4), posibilitando establecer vínculos de cooperación con numerosos grupos que actuaron para la comunidad en escuelas y universidades.

Sin quedar limitada a la enseñanza o restringir sus servicios a los jóvenes, la extensión logró el propósito de actuar como intermediaria para poner en contacto a personas, organizaciones y comunidades tanto con las fuentes de información e instrucción, como con quienes habían logrado un conocimiento experto de ciertos temas. Se esperaba que a través de las modalidades adoptadas "la vida de la ciudad fuese actualizada periódicamente por las corrientes de pensamiento e inspiración del exterior, evitándose el estancamiento mental o la mera absorción por los negocios" (University of Minnesota, 1917:5).

De esta forma, los resultados y trascendencia de la experiencia extensionista durante el transcurso de veinte años fueron identificados como "los signos inconfundibles de un movimiento nacional y espontáneo" (Roberts, 1894:122; Draper, 1923:48) con mayores implicaciones de las que sus impulsores habían previsto. Esto significó asimismo el establecimiento eventual de un nuevo tipo de sistema universitario ${ }^{4}$ y el reconocimiento de que la extensión no debía quedar limitada al rol educador que había tenido en Inglaterra en su origen. No solamente constituía una facilidad ofrecida a las clases medias para completar su educación

4) El afianzamiento de un esquema de afiliación desde 1886 en Inglaterra, al otorgar reconocimiento universitario definitivo a los estudiantes que realizaban estudios de extensión de una duración de tres años, aseguró un grado de continuidad y sistematización que hubiese resultado impracticable sin tal reconocimiento. La experiencia de Cambridge en 1873, basada en el método de enseñanza adoptado, se destacó por haber alcanzado "un éxito por completo" que posibilitó que gran número de ciudades exigiera el acceso a estas actividades (Picavet, 1902). 
general o mantenerse actualizadas sobre el progreso de la ciencia, sino que su práctica en Inglaterra y Francia evidenciaba el afianzamiento de su papel social y político a través de realizaciones significativas como la organización de las Universidades Populares de París y los settlements de Londres (Picavet, 1902:2). Esta óptica dio lugar al debate acerca de la conveniencia o no de que el papel social pudiese sustituir al político y permitió discutir alternativas para conciliarlos.

Hasta inicios del siglo XX prevaleció en la experiencia europea y estadounidense de la extensión universitaria una dimensión orientada en lo esencial a la difusión o divulgación. La misma se estructuró en torno a la trasmisión del conocimiento científico y las manifestaciones artísticas y culturales. Moulton destacó el papel de ideas similares que constituyeron la inspiración del trabajo y de los métodos variables según las localidades, para subrayar que

"la extensión de la universidad inglesa no ha sido un esquema cuidadosamente planeado, sino una hazaña de simetría institucional: producto de un propósito simple, perseguido durante muchos años, en medio de condiciones externas variables, en que cada modificación fue sugerida por las circunstancias y probada por la experiencia". (1891:174-178)

La complejidad de las acciones, los escenarios cambiantes, el interés suscitado en campesinos y trabajadores y el sostén proveniente en particular de los sindicatos y las sociedades cooperativas llevaron a que las ideas que la animaron se volvieran más profundas y progresivamente más audaces. En particular, se destaca el impacto en el movimiento cooperativo que reconoció y afirmó con creciente convicción la importancia de la educación ininterrumpida y sistemática como una condición ineludible para su desarrollo ${ }^{5}$ buscando al mismo tiempo sostener —entre otras- la iniciativa de Stuart en el campo de la extensión universitaria. El propio Stuart había expresado su deseo de una universidad sostenida con la ayuda del movimiento nacional cooperativo al sugerir la creación de una universidad en una carta dirigida al Co-operative News (Barnard, 2013:9) por ser el periódico regular del movimiento cooperativo editado a partir del año 1871.

\section{La experiencia inicial en extensión universitaria en Argentina}

La función de la extensión fue contemplada en el marco de la política universitaria al definirse los objetivos de la Universidad Nacional de La Plata en 1905. Se ha destacado el papel de Joaquín V. González, fundador y rector de esa institución, debido a la atención que le otorgó durante su gestión, entre los años 1906 y 1918, a establecer desde el inicio los lineamientos iniciales de la extensión universitaria, en correspondencia con nuevos puntos de vista que manifestó (Castiñeiras, 1938). Claramente influenciada por el positivismo y basándose en la orientación de filántropos anglosajones y socialistas fabianos dedicados a la promoción de la educación, la extensión subrayó la búsqueda de una enseñanza de carácter científico basada en el modelo de "progreso social". En ella se encuentra la influencia de juristas como

5) Los Pioneros de Rochdale expresaron en su estatuto social de 1844 la preocupación por la educación popular y organizaron, tempranamente, una biblioteca y diversos cursos, y dispusieron, en el artículo 42 del nuevo estatuto social adoptado en 1854, el destino del $2,5 \%$ de los excedentes que obtuviese la sociedad a la formación de un fondo para la educación de los asociados (Kaplan y Drimer, 2017). 
Adolfo Posada en la Universidad de Oviedo, España, en 1898, y Rafael Altamira, quien había expresado el interés por el acercamiento a la clase obrera y la decisión de ejercer sobre ella "una tutela educativa" (2016:266). Ambos consideraban como extensión toda labor de carácter educativo y social realizada por la universidad fuera de su ámbito oficial docente y destacaban que su principal misión no era instruir sino educar, es decir, "elevar el espíritu, abrirle horizontes nuevos, dignificarlo", para poder apreciar los bienes que "compensan la monótona y al cabo embrutecedora repetición de un trabajo mecánico casi invariable" (Altamira, 1997:182). En esta concepción se acentuaba el interés para que la universidad contribuyese a construir la educación popular, rompiese su aislamiento y se comunicara directamente con las clases sociales que no accedían a ella. Con esta orientación, la Universidad de Tucumán también impulsó desde su creación diversas actividades entre las que sobresalieron las de extensión orientadas al medio rural y los productores. ${ }^{6}$

La preocupación de las universidades por desarrollar su acción, más allá de su función académica en la enseñanza y la investigación, fue contemplada formalmente entre los postulados de la Reforma Universitaria de Córdoba de 1918. Desde entonces, la función extensión se ha podido expresar en programas específicos que, por lo general, remiten a la universidad en la profundización de su rol como institución comprometida con la transformación social. Estos programas acercan la producción y la transmisión de conocimiento a sus efectivos destinatarios y cuidan que en ese proceso la apropiación social del conocimiento, de las ciencias y de las tecnologías, sea simétrica y contribuya a salvar la brecha que separa al ciudadano común de la ciencia, dándole así los medios para que se forme una opinión sobre las prácticas y las políticas que afectan su vida cotidiana y pueda participar con más conocimiento y responsabilidad (Vessuri, 2000:89).

Se ha destacado que la misión social de la universidad constituyó el extremo programático de la Reforma Universitaria al agregar a su propósito un nuevo quehacer que permitiese vincularla más estrechamente con la sociedad y sus problemas. Acorde con esta aspiración, la extensión universitaria y la difusión cultural fueron incorporadas a sus tareas corrientes y generaron un conjunto de actividades que se tradujeron en determinados momentos en una mayor concientización y politización de los estudiantes universitarios. Así, se asumieron tareas que no se proponían o que permanecían inéditas para las universidades de otras regiones del mundo (Tünnermann Bernheim, 2000:100).

\section{El papel de la UBA}

Entre las recomendaciones que surgieron de la Reforma con respecto a la extensión en los ámbitos académicos, se destacaba la incorporación en los planes de estudio de una materia de enseñanza con carácter social y el inicio de campañas contra el analfabetismo a ser realizadas por los centros de estudiantes mediante la organización de colegios nocturnos para

6) En la apertura de los cursos de 1915, el rector Juan B. Terán, al considerar que la enseñanza había sido de excepción, aristocrática y cerrada, reivindicó la importancia de la universidad moderna para alcanzar al mayor número con sus beneficios y desde esta perspectiva subrayó que la "extensión universitaria, característica de su nuevo espíritu, educa al adulto y a la mujer". Propuso realizar la extensión agrícola en la Universidad de Tucumán: "irá el profesor a la campaña, a la propia casa del campesino (...) formará parte de ella el servicio permanente de información y la instrucción por correspondencia (Anales de la Facultad de Derecho y Ciencias Sociales, 1915:717). 
obreros - con la colaboración exclusiva de los estudiantes- en los que se impartirían enseñanzas correspondientes a los primeros grados de las escuelas comunes. En esa dirección y apartándose de la línea extensionista impulsada en los años precedentes, se inauguraron en la Facultad de Derecho, en 1920, los primeros cursos regulares de extensión universitaria destinados a obreros y empleados. En un manifiesto explicando su significación se resaltaba, entre otras, la necesidad de la universidad de "ir hacia el pueblo, ilustrarlo y colaborar en la creación de normas que lo protejan contra la violencia y el privilegio" (Sanguinetti, 1956:3), enfatizando en esa línea que la emancipación intelectual de los trabajadores debe ser la obra de los propios trabajadores.

En las sesiones del Consejo Directivo de la Facultad de Derecho se acentuaba la importancia de esta función y se la consideraba promotora de un reconocimiento de la universidad como ámbito que "por no pertenecer ni deberse a una clase determinada, ni ser un mero instrumento del Estado, sino la resultante del medio social, debía responder a un sistema de intercambio y de acciones y reacciones mutuas en el medio social" (Revista de la Facultad de Derecho y Ciencias Sociales, 1925:T. IV no 12, 900).

La actividad principal de la extensión estuvo centrada en el dictado de conferencias, preferentemente por profesores y alumnos de autoridad académica, aunque también se invitó a expositores que contaran con amplio reconocimiento. Si bien no siempre existió consenso con respecto a temas formales y de contenido sobre las actividades que debían promoverse, o a la conducción de la Comisión de Extensión Universitaria, el consejero que la presidió desde su inicio en 1922 destacó el papel de esta función como divulgadora de la enseñanza en los medios no universitarios y especialmente entre los sectores sociales más interesados en la justa solución de los problemas jurídico-económicos (Revista de la Facultad de Derecho y Ciencias Sociales, 1925: T. IV, 902). La comisión que había sido designada al concluir los cursos de 1922 quedó disuelta poco más tarde como consecuencia del cambio en la política universitaria nacional desde 1923, cuando las intervenciones a las casas de estudios se reflejaron en la consecuente modificación de sus estatutos y el avance de los sectores antirreformistas. Sin embargo, conforme a un plan provisorio, ${ }^{7}$ fueron dictadas varias conferencias entre las que se destacó la pronunciada por Alfredo L. Palacios el 6 de noviembre sobre Derecho Internacional Obrero. Al señalar la contradicción existente entre la presunción jurídica de que todos conocen la ley y la propia realidad del conocimiento rudimentario que posee el pueblo de las normas, se subrayó la importancia de divulgar los conocimientos jurídicos, considerando que la extensión universitaria venía a cubrir esta finalidad (Revista de la Facultad de Derecho y Ciencias Sociales, 1922: T. I, 247-248). También se desarrollaron conferencias sobre cooperación dentro y fuera del ámbito de la facultad y la temática fue contemplada por el Dr. Juan B. Justo, quien fue invitado para tratar el tema de la vivienda (Revista de la Facultad de Derecho y Ciencias Sociales, 1925: T. IV [n 12], 880).

7) Para algunos integrantes del Consejo Directivo, la labor de extensión había sido descuidada y expresaba asimismo parcialidad ideológica. Sin embargo, en años previos a la Reforma Universitaria, el decano, Dr. Eduardo L. Bidau, en la inauguración de los cursos de 1916 (Anales de la Facultad de Derecho y Ciencias Sociales, 1916:626), subrayó no haberla descuidado ni haber incurrido en discriminaciones destacando la invitación realizada a expositores nacionales y juristas europeos como Enrico Ferri, Rafael Altamira y Léon Duguit entre 1909 y 1911. Al respecto, se debe destacar la conceptualización jurídica y la actuación pública de Duguit como reflejo de una concepción republicana, laica y social, promotora del reemplazo de la vieja idea de derecho subjetivo por la nueva noción de "función social". 
Las actividades de extensión durante ese período se orientaron a desarrollar sistemáticamente acciones de divulgación cultural de tipo científico a través de conferencias que gradualmente adquirieron un mayor nivel de sistematicidad y un desarrollo de contenidos que les permitieron adoptar la forma de cursos de extensión. Por lo general, se trató de actividades abiertas a la comunidad cuyas temáticas requirieron un nivel de formación en concordancia con el tipo de contenidos que se difundía. Se orientaron a la comunidad universitaria y a los ámbitos sociales de pertenencia de los estudiantes, poniendo de manifiesto la expectativa por el desarrollo científico y el interés en mostrar la utilidad y el aporte de los conocimientos para la sociedad.

Las referencias precedentes evidencian que los impulsores de la extensión trataron de diferenciar la actividad desarrollada en la universidad argentina de aquella inspirada en la concepción clásica europea, aunque prevaleció en los primeros años de la Reforma el propósito de la difusión de conocimientos y cultura universitaria desde la universidad —como transmisora- a la comunidad receptora. Esta perspectiva planteó predominantemente relaciones unidireccionales, aunque persistió entre quienes la propiciaron la convicción de una extensión universitaria que debía ir más allá de los estatutos o de los métodos pedagógicos para ser instituida en su carácter de servicio. En gran medida, sus realizaciones iniciales reflejan la posición y el compromiso de juristas liberales confiados en las virtudes de la instrucción pública, propulsores del papel de la universidad como instrumento de difusión de una cultura laica y defensores del derecho como instrumento necesario para el progreso social y la educación de la clase trabajadora.

\section{El cooperativismo y el quehacer del Departamento de Extensión Universitaria de la UBA}

Desde una perspectiva integradora de las modalidades de extensión prevalecientes en la Universidad desde su origen, con un tipo de actividades que acentuaba la importancia de ampliar y proyectar su acción acercándola a las necesidades sociales, fue creado en 1956 el Departamento de Extensión Universitaria de la Universidad de Buenos Aires (DEUBA). ${ }^{8}$ La iniciativa tendió a concretar una imprecisa expresión de anhelos en torno a "la universidad para el pueblo", consigna que carecía en los años 1950 de una concepción práctica, integral y concreta (Sanguinetti, 1956:58). El postulado desde el que se desarrollaron las actividades del Departamento sostenía que si la universidad quería contribuir a las necesidades del país, debía encarar la solución de los problemas fundamentales, que orientasen y enriqueciesen su tarea, mediante la convergencia de distintos sectores sociales, perspectivas y puntos de vista que integraban la realidad, lo que contribuía a generar mayor conciencia por parte de la población del país (UBA, 1958:4). El primer año de la actividad del Departamento se consagró a concretar los alcances y definir el propósito de integración de la Universidad a su entorno social, con la finalidad de ampliar su acción fuera de los estrechos límites en que se desarrollaba y acercarse concretamente a la vida nacional. La tarea propuesta radicó principalmente en "producir un cambio recíproco entre el hombre de pueblo y el científico y el técnico" (Departamento de Extensión Universitaria, 1956:58) para que el primero contase

8) En 1956 se creó el IEC por resolución del interventor interino de la UBA, designándose una comisión honoraria como encargada de establecer sus objetivos y modos de organización (Res. UBA 73 26/01/1956). 
con los elementos necesarios para la solución de sus problemas y el hombre de ciencia y el técnico asumiesen su responsabilidad en la obra de bien común. La tarea visualizó como inseparable la transformación de las estructuras de la sociedad y las de la Universidad, ya que se trató de un trabajo sostenido por los docentes del Departamento y el conjunto de estudiantes que participaron voluntariamente y fueron capacitados para ese trabajo. La UBA propició así la extensión universitaria como órgano permanente de docencia y de acción social priorizando un plan de trabajo que debía responder en sus temas a la clase obrera de manera exclusiva, preferentemente la ubicada en los barrios industriales y centros obreros de la ciudad y de la provincia de Buenos Aires.

Desde esta perspectiva, las actividades de extensión fueron concebidas con la finalidad de permitir vincular o revincular al estudiante y al docente, y por ende a su institución, con las problemáticas nacionales propias así como con aquellos ámbitos de la misma Universidad (en especial facultades y cátedras) que anteriormente habían estado desvinculados. Las actividades debían ser, en lo primordial, facilitadoras de una comunicación intra e interinstitucional. Al respecto, cooperativistas como Juan B. Justo y Nicolás Repetto ya habían subrayado el papel de la extensión al mencionar la necesidad de familiarizar al pueblo para comprender los problemas de su tiempo y ayudar a resolverlos, advirtiendo así la verdadera naturaleza de esta función. Asimismo, había sido justificada su necesidad debido a que la enseñanza en cualquier grado es "una actividad propia del Estado, un servicio público que debe beneficiar en mayor o menor intensidad no solo a los destinatarios inmediatos, sino y en forma más limitada y mediata, a la comunidad" (Bielsa, 1928:41).

Cuando el programa tuvo inicio sobresalieron las actividades desarrolladas a partir de 1956 en el Centro Integral de Isla Maciel con los vecinos de un barrio integrado por los sectores de la Isla Maciel y la Villa Maciel. ${ }^{9}$ En ellos habitaba una población obrera dividida en zonas separadas: una con viviendas humildes, formada por familias originarias del Gran Buenos Aires o por antiguos inmigrados, y otra con casillas de emergencia habitadas por inmigrantes originarios de provincias del interior. La búsqueda de respuesta a las demandas y necesidades de ambos sectores facilitó el trabajo de extensión, que también se interesó por suprimir las causas de la segregación que se producía entre las dos zonas.

En diez años de trabajo se estudiaron "hombro a hombro", junto con los pobladores de la isla, las soluciones para los problemas del barrio (UBA, 1966:3) para poder delimitar tres tipos de proyectos: el Centro de Desarrollo Integral, un programa de comunicación a través de la realización de ciclos radiales y la reactivación de la Biblioteca Popular "Almafuerte" de Sáenz Peña, en el Partido de San Martín. Una perspectiva pedagógica guiada por la aplicación del sistema de "Educación Fundamental" se orientaba al asesoramiento técnico de la población para la solución de sus problemas básicos, estimulando las iniciativas e impulsando la participación.

9) Isla Maciel es un barrio de Dock Sud, en Avellaneda, situado en el límite sur de la ciudad de Buenos Aires, fundado en 1887. Los residentes más antiguos eran descendientes de inmigrantes genoveses dedicados al oficio de construcción naval y peones industriales provenientes del interior del país. Constituyeron el sector de la Isla Maciel construyendo casas de chapa acanalada y madera, dispuestas bajo la forma de conventillo. A principios del siglo XX, los astilleros, frigoríficos y curtiembres fueron los principales ámbitos de ocupación para la población de la isla. Por su parte, los residentes de la Villa Maciel tenían origen en los trabajadores golondrina, provenientes del litoral, que se asentaron en terrenos fiscales, carentes de servicios. 
De esta forma se logró dar respuesta a las necesidades de la población escolar a través de un programa educativo que incluyó una escuela vespertina en cuyos cursos se trató de responder a las necesidades locales expuestas por los representantes vecinales; un centro de recreación, un centro juvenil y un jardín de infantes que posibilitaron que, en el curso de diez años, de cada diez niños en edad escolar, siete completaran la escuela primaria. Por su parte, en el centro de salud se desarrolló un programa de medicina preventiva basado en un estudio realizado por el Departamento de Extensión Universitaria de la Facultad de Ciencias Médicas y la cátedra de Medicina e Higiene Social.

Además del mejoramiento de cada sector y de los proyectos educativos y de salud, los vecinos decidieron asociarse para conformar una cooperativa de consumo ya que encontraron problemas concernientes por igual a los habitantes de la isla y a los de la villa. Un grupo de socios con experiencia en el trabajo en cooperativas fue visitando a las familias para explicar los principios de la cooperación y, en la Navidad de 1957, en una casilla donada por la Municipalidad de Avellaneda, comenzó a funcionar la Cooperativa de Consumo Isla Maciel. Los miembros del Consejo de Administración y los socios más activos trabajaron, entre otras actividades, en la atención al público, la búsqueda de productos directamente de fábrica y las tareas de difusión. La finalidad del trabajo era impedir que los precios de los productos subiesen "más de la cuenta", de modo tal que, cuando la cooperativa ya estaba funcionando, se fortaleció su papel en la comunidad por tratarse de una organización que había puesto "un freno a la especulación", y llegó a contar —en 1966 — con cerca de 500 socios (UBA:11).

La gestión de las tareas realizadas en el Centro de Desarrollo Integral Isla Maciel estuvo a cargo de un Consejo Administrativo integrado por representantes de varias organizaciones barriales, quienes definieron el estatuto y sus modalidades de funcionamiento. En los estatutos aprobados estaba prevista la integración del Consejo Directivo por un secretario general elegido obligatoriamente entre los representantes vecinales, un prosecretario perteneciente al DEUBA, dos secretarios de prensa y propaganda, dos de finanzas (uno elegido entre los universitarios) y un secretario de actas. Se estableció que los cargos no fueran desempeñados por las personas delegadas sino por las instituciones, y en ellas recayó la responsabilidad de las funciones. Los representantes de las facultades cuyas cátedras participaban en el proyecto también integraban el Consejo Directivo aunque su número debía ser inferior al de los delegados vecinales. ${ }^{10}$

La actividad de extensión reflejó las situaciones históricas, sociales y políticas de la sociedad argentina de la época del reformismo frente al esquema de la revolución que comenzaba a aparecer como alternativa y también la influencia de corrientes de la derecha. Sin embargo, en el DEUBA el debate ideológico no se daba internamente,

"era un grupo bastante homogéneo, el que entraba a trabajar ahí en algún punto aceptaba las reglas de juego del proyecto, no lo podías cuestionar porque si no ¿para qué estabas ahí? Era bastante voluntario el trabajo, a pesar de que había gente voluntaria y había gente rentada, pero si estabas ahí era por voluntad propia". (Tedesco, 2011, cit. por Diamant y Urrutia, 2013)

10) Entre las actividades para adultos realizadas en el primer año, el encuentro sobre el cooperativismo contó con la participación de los socios de las dos cooperativas de la Villa Maciel y de personas de otras instituciones de la zona que permitieron que el Instituto de Estudios Cooperativos pudiese programar un curso sobre el tema. 
Desde esa perspectiva, la incorporación de la misión social de la Universidad junto con las funciones tradicionales de preservación del patrimonio cultural, investigación científica y formación de profesionales, aspiraba a convertir la institución en uno de los factores principales del cambio social, dando respuesta a las necesidades, requerimientos y problemas de la sociedad para contribuir al desarrollo de la comunidad (Frondizi, 1971). El Departamento debía centrarse en concretar esta misión y para ello era indispensable el contacto permanente con la sociedad, en especial con los sectores más desfavorecidos, de modo de identificar sus problemáticas y dar respuesta a las mismas.

Con esos supuestos, el desarrollo de acciones concretas produjo un avance cualitativo institucional en el ámbito de la extensión como resultado de un conjunto de innovaciones que explican la importancia de las actividades orientadas a los sectores populares urbanos. A ellas se incorporaron acciones académicas que se constituyeron en puntos clave del proyecto de reforma y modernización universitaria, albergando a estudiantes y graduados de casi todas las facultades de la UBA. En su mayoría, se trató de un conjunto de docentes provenientes de las ciencias sociales entre los que prevalecía la idea de la educación permanente, la educación para el progreso social basada en el asesoramiento técnico a la población para la solución de sus problemas básicos, el estímulo a la iniciativa espontánea, la acción directa con las personas (DEUBA, s/f) y la intervención orientativa no prescriptiva.

El advenimiento del gobierno militar de fines de julio de 1966 y el quiebre del orden institucional desencadenaron la intervención de la Universidad, la derogación de la Ley Universitaria y la vulneración de la autonomía y el gobierno universitario tripartito. Esto condujo a que quienes trabajaban en el Departamento renunciasen a sus puestos de trabajo por no sentirse comprometidos con una "universidad cerrada, ciega y muda (...) ni cómplices de una institución donde hablar de los problemas del pueblo se convierta en delito de opinión" (UBA, 1966:31)

No obstante, el alcance de la experiencia fue tal que se ha podido rescatar en su contenido y sus prácticas la noción de la universidad y de la extensión universitaria como un "servicio público" concebido como "una función social definida en torno a la apertura hacia el pueblo y en servicio del pueblo" (Sarlo, 2001:75). Su principal contribución radicó en proyectar un marco de referencia preciso sobre la naturaleza de la extensión universitaria en un campo específico. Permitió poner de manifiesto la potencialidad de una práctica colectiva que, además de identificar los intereses y aspiraciones de un grupo, pudo explicar las razones de su compromiso con la Universidad y con organizaciones como las cooperativas, planteando reflexiones que favorecieron una visión a largo plazo. Aunque la experiencia no constituyó una evolución lineal, las estrategias adoptadas de manera colectiva posibilitaron avanzar en un campo de incertidumbre y edificar progresivamente confianza y certezas.

\section{El papel de la Facultad de Ciencias Económicas de la UBA}

En la línea de realizaciones del DEUBA, el 29 de julio de 1957 fue creado, en la Facultad de Ciencias Económicas, el Instituto de Estudios Cooperativos (IEC), ${ }^{11}$ vinculado con la cátedra

11) Su creación mediante Resolución № 707 se inscribe en las disposiciones del Reglamento del Departamento de Extensión Universitaria de la FCE creado el 05/11/1956 por Res. № 408, durante la gestión del decano interventor Isidro Martínez. 
de Sociedades Anónimas, Seguros y Cooperativas. La cátedra había incluido desde 1958 temáticas específicas relacionadas con las cooperativas para hacer hincapié y diferenciar la naturaleza y características de estas empresas enfatizando la importancia de su régimen legal y los atributos de los distintos tipos societarios. ${ }^{12}$

La creación del Instituto respondió a la necesidad de ampliar el campo de las investigaciones en las disciplinas de especialidad de la facultad y preparar mejor a sus egresados, al tiempo que aportaba conocimientos de valor sobre la realidad económica nacional a través del estudio de los problemas más destacados. Asimismo, buscó realizar estudios especiales sobre el desarrollo de las cooperativas del país justificados por el grado de trascendencia que estas organizaciones presentaban en el campo económico y social. Cabe señalar que, al momento de la creación del Instituto, ya se habían constituido en el país 2780 sociedades cooperativas que contaban con más de un millón de asociados (Dirección Nacional de Cooperativas, 1964:18).

La reglamentación del IEC mencionaba, junto con su función desde el punto de vista docente, la necesidad de focalizar los estudios en problemas vinculados al cooperativismo, con especial referencia a la situación económico financiera y social nacional, para destacar entre sus objetivos la necesidad de complementar la enseñanza con la práctica de la investigación científica sobre la materia. Se aludía a la responsabilidad para atender las consultas que se le formulasen sobre cooperativismo - por intermedio del Decanato- en cuanto a aspectos particulares del mismo y asuntos o problemas precisos. La actividad debía generar y mantener una permanente vinculación con las cooperativas y las entidades y estudiosos que sostuviesen principios análogos dentro y fuera del país. Se consideraba que la enseñanza teórica y la práctica complementaria que se impartía en la cátedra de Sociedades Anónimas, Seguros y Cooperativas estaban inspiradas en el principio de "aprender haciendo", por lo que su interés era el de formar a los estudiantes para enfrentar las situaciones novedosas, dar respuestas a las carencias que ofrecía la realidad y promover durante su formación la toma de conciencia social de sus obligaciones para con la sociedad en la que estaba inserto.

Guiado por el interés en contribuir a mejorar las condiciones de vida de las "clases laboriosas y dar soluciones prácticas a los problemas generales del país" (Aldrey, 1958:130), el IEC acompañó el trabajo de extensión impulsado por la UBA para responder a necesidades sentidas y a los deseos manifiestos por los pobladores de Isla Maciel, en particular en la constitución y desarrollo de experiencias cooperativas de consumo y de vivienda. ${ }^{13}$

12) La extensión tuvo un espacio importante en la enseñanza universitaria de la Facultad de Ciencias Económicas, donde Domingo Bórea dictó un curso optativo sobre cooperación entre 1924 y 1926 y en 1931. Este especialista se incorporó en 1912 al Ministerio de Agricultura de la Nación como jefe de Economía Rural. Desde su función colaboró con la realización del tercer Censo Nacional levantado el $1^{\circ}$ de junio de 1914, bajo la presidencia de Roque Saenz Peña. En volumen separado del mismo publicó una monografía sobre la mutualidad y el cooperativismo en la República Argentina donde resume la importancia de estos movimientos en Argentina que en 1914 contaban con 600 000 mutualistas y cooperativistas (7,5 \% del total de la población nacional) agrupados en 1301 sociedades. Bórea colaboró con la Comisión de Códigos del Senado de la Nación que sancionó en el mismo año la Ley 11.388 sobre "Régimen Legal de las Sociedades Cooperativas", primera norma nacional sobre cooperativas de nuestro país, y la Ley de "Fomento de la Cooperación". En virtud de la promulgación de ambas leyes, en 1927 se creó el Registro Nacional de Cooperativas dependiente del Ministerio de Agricultura. La organización del mismo y su dirección fueron confiadas al Dr. Domingo Bórea, quien a partir de entonces comenzó una tarea constante y fructífera, asesorando a cooperativas y participando de las inquietudes de sus dirigentes (Bórea, 1917).

13) Cabe destacar la implicación y compromiso de Alicia Kaplan de Drimer — profesora del IEC_- por su trabajo en la organización de las entidades y por su apoyo a las actividades del DEUBA. 


\section{La constitución de cooperativas y las necesidades de los pobladores de Isla Maciel}

El programa de extensión denominado "Organización de Comunidad" destacó la necesidad de realizar avances a partir de las instituciones de fomento barriales para coincidir en acuerdos sobre los problemas identificados como más importantes para el barrio y determinar el orden en que debían resolverse. Se trataba de alcanzar progresos "paso a paso" y duraderos. Este recorrido resaltaba la búsqueda de un mejoramiento integral, extensivo a todos, que incluía también aspectos materiales y sociales ${ }^{14}$ (UBA, 1966:9).

Así surgió la Cooperativa de Consumos Isla Maciel Ltda., ante la urgencia de mitigar o resolver las consecuencias "nefastas que para el presupuesto del hogar tiene el alza operada en el costo de la vida, y considerar su papel para mejorar el poder adquisitivo de sueldos y salarios" (Aldrey, 1958:132). Su objetivo fue la adquisición de productos o artículos en común para su distribución a precios reducidos entre sus asociados, retornando los excedentes resultantes al finalizar cada ejercicio en proporción a las compras efectuadas. ${ }^{15}$

La Cooperativa de Vivienda de Villa Maciel Ltda., por su parte, fue consecuencia del interés por resolver de manera progresiva la situación que generaba el inminente desalojo, por parte de la Empresa de Ferrocarriles del Estado Argentino (EFEA), de más de 100 vecinos de la villa de los terrenos fiscales que ocupaban. En el marco del proyecto, se iniciaron en 1960 los estudios para el desarrollo de la construcción de una unidad vecinal por etapas ${ }^{16}$ en función de las características de la población destinataria de los edificios (Gaite, 2007:27, 220-235). En la opción de diseño de casa colectiva prevaleció la concepción del arquitecto Wladimiro Acosta, al tomar en cuenta las condiciones de habitabilidad de los pobladores en sus lugares de origen y considerar que la construcción de la vivienda debía ser

"solo una parte de un amplio y profundo trabajo de adaptación a un nuevo medio social y a una nueva manera de vivir (...) con ella deben ir la educación, el estímulo a la formación de nuevos hábitos de vida, la creación de una conciencia sanitaria y social, entre otras metas" (Acosta, 1984:80).

En tanto, los miembros del IEC acompañaron las gestiones de los vecinos desde el inicio de la conformación de una comisión interesada en buscar rápidas soluciones con la creación de una cooperativa de vivienda que adoptó la decisión de que las unidades construidas fueran propiedad de la entidad, otorgando a los socios el derecho de uso sobre la unidad adjudicada cuando esta se concluyese.

14) En el balance de realizaciones se ha destacado la instalación de piletas de lavar, la extensión de redes de agua corriente y luz eléctrica, el relleno de pasillos, la construcción de locales para las instituciones y el parque infantil y deportivo a los que se agregan las acciones solidarias frente a incendios, amenazas de desalojo o inundaciones.

15) Asimismo, se trató de solucionar en forma cooperativa, entre personas de escasos recursos, el problema de la conservación de los alimentos mediante un pequeño frigorífico de uso común reservando espacios individuales a cada socio. Una cuota reducida para contribuir al funcionamiento de un servicio que además de contribuir a que se evitaran pérdidas de productos incidió en la disminución de los gastos familiares en la alimentación.

16) El equipo de extensión del proyecto estaba constituido por cinco alumnos de los últimos cursos que participaban de un taller dirigido por el arquitecto W. Acosta en la Facultad de Arquitectura y Urbanismo (Acosta, 1984:79). La cooperativa de Vivienda "Villa Maciel Ltda." se constituyó definitivamente el 8 de diciembre de 1957 con el apoyo del Instituto de la Vivienda de la Provincia de Buenos Aires. El Estado nacional autorizó la cesión de terrenos a la cooperativa por Ley 14.861 del 15/09/1959 y el Banco de la Provincia de Buenos Aires dispuso de un fondo de 125 millones de pesos para otorgar créditos para la compra de viviendas familiares por plazos mayores de 35 años, de hasta el 90 $\%$ de la tasación conjunta del terreno y el edificio con un interés anual de 2,5\%. 
Los procesos de formalización y el desarrollo de las actividades en las cooperativas fueron sostenidos por un grupo de alumnos del Instituto apoyados por su director y docentes que "enseñaron con todo empeño y diligencia" (Aldrey, 1958:133) los principios requeridos para su funcionamiento y prestaron el asesoramiento necesario. Según los partícipes de la experiencia -docentes, estudiantes y pobladores—, la organización de las actividades permitió integrar iniciativas individuales con momentos colaborativos de trabajo. Así, en la cooperativa de consumo, las actividades individuales se realizaron para el registro de productos para la venta, la compra de insumos, la organización y disposición de productos del almacén, el seguimiento de stocks y ventas, el control de los excedentes y el cálculo de su reintegro, mientras que en la de vivienda se relacionaron con la búsqueda de terrenos para la edificación de las casas, el contacto con organismos públicos y bancos, el acercamiento a otros vecinos o el registro y el cobro de la cuota social. De manera complementaria, el trabajo con otros fue particularmente extendido y se expresó en la tarea voluntaria en los consejos de administración, en la asistencia y participación en las asambleas, en la organización de cursos y en la discusión de propuestas e iniciativas.

El balance de la acción realizada permitió a los docentes de la FCE actuar como mediadores al estimular los aprendizajes y, en especial, al favorecer el desarrollo de potencialidades en torno al respeto por la necesidad del otro, sin dejar de subrayar el acompañamiento necesario para construir "una capacidad de liderazgo efectivo y global" (Erbin, 2002: Anexo, 5-7) especialmente cuando se trató de asumir responsabilidades en cargos de conducción.

En síntesis, los aportes de esta experiencia posibilitan destacar la originalidad de una iniciativa que admitió avanzar en direcciones paralelas y complementarias profundizando en un conjunto de prácticas que favorecieron la adecuación y el ajuste de las propuestas a las características de la población y al contexto en el que se trabajó, la búsqueda de autonomía de las cooperativas creadas y el impulso de experiencias que conciliaron la heterogeneidad de necesidades y posiciones.

De esto se desprende la valoración del cooperativismo, concebido por el programa como un fin en sí mismo y no solo como un medio para lograr otros objetivos, al entender su papel para familiarizar al pueblo con sus problemas y ayudar a resolverlos. Esta orientación fue coincidente con la perspectiva de hacer de la extensión "un servicio público de sentido social, destinado exclusivamente a las clases laboriosas" (Sanguinetti, 1956:2-4). Al recuperar las prácticas e ideas de los fundadores del pensamiento cooperativo en Argentina y resaltar el valor de la cooperación libre ${ }^{17}$ se fortaleció la confianza en la cooperativa como un tipo societario ideado para poner en manos exclusivas de los productores o de los consumidores el manejo de sus intereses.

Igualmente, cabe notar que la posibilidad de superar las limitaciones requirió un trabajo integrado y coordinado que propició la asociación y creación de comunidades de aprendizaje mutuo con las organizaciones a las que la extensión se orientó, facilitando el cumplimiento del papel académico y educativo propio de la universidad. Al respecto, es necesario destacar la intencionalidad transformadora de la experiencia de extensión analizada, en la

17) Se trata de figuras relevantes del partido socialista vinculadas a la cooperación, como Juan $B$. Justo, quien expone en 1909 los principios de la cooperación libre (1977), y Nicolás Repetto. Específicamente, la Cooperativa de Consumo, Edificación y Crédito Ltda. había sido fundada por Juan B. Justo el 30 de julio de 1905 bajo el lema "La cooperación libre es la solidaridad para hacer". 
medida en que los actores sociales fueron reconocidos como sujetos protagonistas de ella y no como mero objeto de las intervenciones universitarias.

\section{A modo de conclusión}

Las experiencias mencionadas en el texto permiten reflexionar sobre el alcance de dos modalidades de extensión universitaria construidas a partir de estrategias diversas y acciones diferenciadas en cuanto a los contenidos y componentes pedagógicos relacionados con el cooperativismo.

Una primera modalidad consiste en introducir acciones educativas — centradas en la temática legal, financiera, impositiva, contable, laboral y societaria - vinculadas con contenidos sobre cooperativismo, paralelas a la programación formal y "al costado" de las actividades universitarias tradicionales. Se trata entonces de acciones reservadas a ciertos tipos de población, en particular estudiantes y eventualmente profesionales egresados y docentes, con el propósito de extender la transmisión de contenidos educativos a poblaciones adultas o grupos activistas de los centros estudiantiles u ofrecer contenidos que se solapan con los contenidos tradicionales de las materias que forman parte de la currícula.

Esta modalidad, que puede caracterizarse como adaptativa, tiende a marginar el proceso de aceptación e incorporación plena de la función extensión requerida en este campo. Es el caso, por ejemplo, del desarrollo de cursos cuya existencia parece ser solo "tolerada", sin ser plenamente reconocida, dado el carácter que se le asigna al curso como materia optativa, por ejemplo, y sin estar concebido como un tipo específico de actividad de extensión. Esta modalidad dual, que puede hacerse extensiva a otros campos, contribuye a proteger al sistema universitario que formalmente estima necesarias las prácticas de extensión y adhiere a ellas, aunque sin reconocer plenamente el valor de un servicio específico necesario para la formación de sus estudiantes o el alcance de las prácticas extensionistas en las propias cooperativas.

La segunda modalidad, caracterizada como implicada, refleja la naturaleza del programa realizado por el DEUBA entre los años 1956 y 1966 y en particular la acción del IEC de la Facultad de Ciencias Económicas. Se trató de una experiencia cuyo potencial radicó en promover una transformación sistémica que permitió equilibrar la perspectiva extensionista en el sistema universitario a nivel de estructuras, programas y métodos, y que repercutió en los objetivos generales de la institución. Muy pocos desarrollos de extensión universitaria alcanzaron en la última década resultados tan amplios y ninguno ha podido reflejarse en una articulación de la enseñanza, investigación y extensión, que se tradujera en el contenido curricular, las relaciones pedagógicas, los criterios de validación del trabajo docente y los temas de la investigación básica y aplicada. Concretamente, la contribución de la Facultad de Ciencias Económicas y su trabajo con las cooperativas de la Isla Maciel, aunque dejase de tener continuidad institucional en 1966, lograron expresar, entre otros cambios, que la educación promovida a través de la extensión —en lugar de considerarse como propia de determinados sectores sociales o períodos particulares de vida- debe ser reconocida como un interés general y permanente. También posibilitaron valorizar el ejercicio del trabajo docente y estudiantil en el barrio e introducir innovaciones relevantes para las prácticas de la institución, las disciplinas involucradas, los métodos de enseñanza y la propia organización 
cooperativa. Específicamente, el trabajo cotidiano en las cooperativas creadas, además de promover cambios en las estructuras establecidas, brindó oportunidades tanto para la adquisición de competencias ciudadanas como para su ejercicio. En este ámbito, sobresalen las prácticas de participación en la cooperativa que favorecieron la construcción grupal de reglas, los vínculos institucionales, la responsabilidad por el cuidado de espacios comunes, el reconocimiento de las diferencias, la consideración por los otros, la toma de decisiones compartidas, los distintos tipos de relaciones establecidas y, en general, la naturaleza de los procesos de convivencia.

Apreciada en conjunto la perspectiva de extensión desarrollada en el ámbito del cooperativismo, permitió articular asimismo las demandas provenientes de distintas organizaciones y actores tanto con la formación como con la investigación, lo cual favoreció un trabajo de reajuste permanente.

Ante estas alternativas, y a modo de conclusión, ¿es posible afirmar qué tipo de objetivos de extensión tienen mayores posibilidades de éxito dependiendo de la adopción de una u otra modalidad? Si bien es muy difícil responder de manera concluyente debido a la especificidad de las situaciones y contextos, en cualquier caso es posible indicar que la segunda estrategia tiende a un cambio puntual e implica una modificación delimitada, más fácil de alcanzar y con potencialidad para asegurar la coherencia interna de cada práctica de extensión y sus diferencias de perspectiva. Aunque los contextos institucionales, locales o nacionales, en los que se adoptan estas estrategias pueden ser muy diferentes, es indudable que las prácticas activas implicadas tienden a modificar desigualdades concretas y favorecer los acercamientos a distintos medios sociales a través de medidas específicas. Desde esta óptica, resulta clave concebir los programas de extensión a partir de las organizaciones y sus problemáticas reconociendo que desde ellas deben partir las iniciativas de construcción de demanda como también la selección de las herramientas más adecuadas a sus necesidades y el estímulo de liderazgos que puedan acompañar el desarrollo y seguimiento del proceso en su conjunto.

La experiencia analizada en el texto pone en evidencia que una tarea de extensión eficaz por parte de la Universidad debe permitir ampliar su mirada institucional y su ámbito específico para ir más allá de la transmisión de conocimientos y poder también construir comunidades de prácticas sostenidas por aprendizajes recíprocos basados en lazos sólidos con el medio, sus actores e instituciones. Esto requiere una reflexión sobre el tipo de herramientas y métodos más adecuados para sostener el diálogo y fortalecer un tipo de conocimiento basado en la complementariedad entre saberes de distinto tipo como recurso indispensable para avanzar hacia una concepción más horizontal de la extensión y promover los vínculos más apropiados para que se puedan beneficiar mutuamente los actores concernidos.

\section{Referencias bibliográficas}

Acosta, W. (1984). Vivienda y Clima. Buenos Aires: Nueva Visión.

Aldrey, M. (1958). Comentarios de Institutos. Realizaciones prácticas auspiciadas por el Departamento de Extensión Universitaria de la Universidad de Buenos Aires en la difusión del cooperativismo entre los pobladores de la isla Maciel. Revista de Ciencias Económicas, Colegio de Graduados en Ciencias Económicas, Centro de Estudiantes de Ciencias Económicas, 46(1, enero-marzo).

Altamira, R. (1997). Psicología del pueblo español. Madrid: Biblioteca Nueva. 
(2016). El patriotismo y la Universidad. Boletín de la Institución Libre de Enseñanza, XXII(462). Madrid. Recuperado de http://www.cervantesvirtual.com/nd/ark:/59851/bmcm34s7 (09/05/2018).

Anales de la Facultad de Derecho y Ciencias Sociales (1915). Discurso del rector de la Universidad de Tucumán doctor Juan B. Terán, en la apertura de los cursos de 1915. Tomo V (1ra. parte, 2da. serie), 710.

—_ (1916). Discurso del doctor Eduardo L Bidau en la inauguración de los cursos de 1916. T. II (3ra. serie), 626.

American Society for the Extension of University Teaching (1890). Address of Richard G. Moulton: on the university extension movement. Recuperado de https://babel.hathitrust.org/cgi/pt?id=umn.31951000811039j;vi ew=1up;seq=10 (09/05/2018).

- (1891). University extension: its definition, history, system of teaching and organization. Philadelphia.

(1900). University Extension Chronology en Ten Years' Report of the American Society for the Extension of University Teaching 1890-1900. Recuperado de https://babel.hathitrust.org/cgi/pt?id=uc1.\$b31156;view= 1up;seq=15 (09/05/2018).

Barnard, P. (2013). Prof. James Stuart and the Rochdale Pionners: How Astronomy as food for tohugt became a Co-op dividend. Antiquarian Astronomer, (7), 4-10.

Bielsa, R. (1928). Cuestiones Universitarias. Política y cultura, administración y jurisdicción. Buenos Aires: Lejouane y Cía.

Bórea, D. (1917). La mutualidad y el cooperativismo en la República Argentina. Buenos Aires: Rosso.

Castiñeiras, J.R. (1938). Historia de la Universidad de La Plata. T. 2 (p. 380). La Plata: Universidad Nacional de La Plata.

Consejo Profesional de Arquitectura y Urbanismo (2018). Proyecto Isla Maciel. Recuperado de https://www. modernabuenosaires.org/proyectosurbanos/proyecto-isla-maciel (09/05/2018).

Departamento de Extensión Universitaria. Universidad de Buenos Aires (DEUBA) (s/f). Primer año de extensión universitaria. Recuperado de http://www.uba.ar/50nbl/archivos/TEXTO\%202.pdf (09/05/2018).

Diez años de extensión universitaria en la Isla Maciel (1966). Cartilla de ex miembros del Departamento de Extensión Universitaria de la Universidad de Buenos Aires dirigida a los pobladores de la Isla Maciel. Recuperado de http://www.uba.ar/50nbl/libros.php (09/05/2018).

Diamant, A.; Urrutia, J.P. (2013). Programas Educativos en el Centro de Desarrollo Integral de Isla Maciel. Voces que visibilizan una experiencia de extensión universitaria en la UBA de los '60. Historia de la educación, 14(2). Anuario.

Dirección Nacional de Cooperativas (1964). Síntesis Estadística de las Sociedades Cooperativas. Departamento de Registro.

Draper, W.H. (1923). University Extension: A Survey of Fifty Years, 1873-1923. Cambridge: Cambridge University Press. Recuperado de: https://archive.org/details/universityextens013150mbp (02/05/2018).

Erbin, B. (2002). Memorias e imágenes de Extensión Universitaria en Isla Maciel. Tesis de Licenciatura. Universidad de Buenos Aires.

Frondizi, R. (1971). La universidad en un mundo de tensiones: misión de las universidades en América Latina. Buenos Aires: Paidós.

Gaite, A. (2007). Wladimiro Acosta. Buenos Aires: Miniediciones Nobuko.

James, E. (1895). Review of the work of the American society for the extension of university teaching, for the four years 1891-1894. Recuperado de: https://archive.org/stream/reviewofworkofam00jame/reviewofworkofam00jame_djvu.txt (09/05/2018).

Justo, J.B. (1977). La cooperacion libre. Buenos Aires: Ediciones Intercoop. 
Kaplan, A. y Drimer, B. (2017). Las cooperativas. Fundamentos, historia, doctrina. 4ta. edición. Buenos Aires: Ediciones Intercoop.

Leclerc, M. (1892). Le rôle social des universités. Paris: Armand Collin et Cie. Recuperado de: https://archive. org/details/lerlesocialdesu00leclgoog (02/05/2018).

— (1894). Les professions et la société en Angleterre: l'éducation et la société en Angleterre. Paris: Armand Collin et Cie. Recuperado de http://gallica.bnf.fr/ark:/12148/bpt6k5509521w/f10.image.textelmage (02/05/2018).

Moulton, R. (1891). University Extension and the University of the Future. Science, 17(425).

Picavet, F. (1902). Introduction, rapports préparatoires, communications et discussions en Troisième congrès international d'enseignement supérieur, tenu à Paris du 30 juillet au 4 août 1900. Paris: Librairie Marescq Ainé. Recuperado de http://gallica.bnf.fr/ark:/12148/bpt6k943478.r=extension\%20universitaire?rk=214593;2 (09/05/2018). Repetto, N. (1958). Mi paso por la política. Buenos Aires: Santiago Rueda Editor.

Revista de la Facultad de Derecho y Ciencias Sociales (1922). Extensión Universitaria. Conferencia del doctor Palacios. T. I, Buenos Aires. Recuperado de http://www.derecho.uba.ar/publicaciones/revista-de-la-facultad-de-derecho/libros/tomo-1.pdf (09/05/2018).

(1925). Sesiones del Consejo Directivo. Sesión del 17 de septiembre de 1924. Tomo IV nº 12, Buenos Aires. Recuperado de http://www.derecho.uba.ar/publicaciones/revista-de-la-facultad-de-derecho/libros/ tomo-4.pdf (09/05/2018).

Roberts, R.D. (1894). Eighteen years of University Extension. London: Blackie \& Son.

Sanguinetti, F. (1956). La extensión Universitaria. Revista del Mar Dulce, I/(5), 1-34.

Sarlo, B. (2001). La batalla de las ideas (1943-1973). Buenos Aires: Ariel.

Stuart, J. (1871). A letter on university extension, addressed to the resident members of the University of Cambridge. Trinity College 23rd November.

Tünnermann Bernheim, C. (2000). El nuevo concepto de la extensión universitaria y difusión cultural y su relación con las políticas de desarrollo cultural en América Latina. Anuario de Estudios Centroamericanos, 4, 93-126.

Universidad de Buenos Aires (UBA) (1966). Usted y la Extensión Universitaria. Buenos Aires: Publicaciones del Departamento de Extensión Universitaria. Universidad de Buenos Aires.

University of Minnesota (1917). University extension: what and why. General extension división, Minneapolis. Recuperado de https://babel.hathitrust.org/cgi/pt?id=hvd.32044079819371 (12/05/2018).

Vessuri, H. (2000). Prioridades de ciencia, tecnología y contexto político. Interciencia, 27(2), 88-92.

\section{Leyes}

Ley 11.388 (1926). Boletín Oficial, 27 de diciembre.

Ley 14.861 (1959). Honorable Congreso de la Nación Argentina. 